\title{
BIOÉTICA EN LA MALLA CURRICULAR DE ODONTOLOGÍA
}

\begin{abstract}
Rina Ana Guerra*
Resumen: El propósito de este trabajo es sondear la situación actual de la bioética como asignatura en el currículo de formación de grado de profesionales odontólogos en Argentina. También se presentan las características del perfil del egresado acorde con las exigencias tecnocientíficas, sociales y culturales actuales y, a partir de ello, se justifica la inclusión de la bioética en el currículo universitario como elemento formador para lograr dicho perfil.

El objetivo último es hacer llamar la atención sobre la necesidad de tener, en la formación del odontólogo, un espacio de reflexión, donde la bioética, llamada ciencia del nuevo milenio, podría ser la opción más adecuada.

Palabras clave: bioética, odontología, universidades, Argentina

\section{BIOETHICS IN ODONTOLOGY'S CURRICULAR NET}

Abstract: This paper's purpose is to explore the present state of bioethics as a subject in odontologists' training curriculum in Argentina. It also shows the graduate's profile and his needs according to techno-scientific, social and cultural requirements that justify the inclusion of bioethics in the university's curriculum. Similarly, it draws attention upon the need of a space for bioethics, the so-called science of the new millennium.
\end{abstract}

Key words: bioethics, odontology, universities, Argentina

\section{BIOÉTICA NO CURRICULUM DE ODONTOLOGIA}

Resumo: O objetivo deste trabalho é de levantar a situação atual da bioética como disciplina no currículo de formação de graduação de profissionais odontólogos na Argentina. São apresentadas atambém as características do perfil do egresso segundo as exigências tecnocientíficas, sociais e culturais atuais e a partir deste referencial, se justifica a inclusão da bioética no currículo universitário como elemento formador para alcançar tal perfil.

O objetivo último é de chamar a atenção a respeito da necessidade de ter na formação do odontólogo um espaço de reflexão, em que a bioética, chamada de ciencia do novo milenio, poderia ser a opção mais adequada.

Palavras chave: bioética, odontologia, universidades, Argentina

* Odontóloga. Especialista en Ortopedia Maxilar y Ortodoncia. Alumna de la Maestría de Bioética, Facultad de Ciencias Médicas, Universidad Nacional de Cuyo, Argentina, 2004-2005

Correspondencia: rinaguerra@ciudad.com.ar 


\section{Introducción}

La esencia de la bioética como disciplina debemos buscarla en la concepción de Potter, oncólogo norteamericano(1), que advirtió sobre el peligro que corría la supervivencia de todo el ecosistema por la ruptura entre el saber científico y el humanístico. Este concepto fue desarrollándose hasta llegar a generar un producto transdisciplinario(2), expresado en la primera gran tarea colaborativa que logró definir a la bioética en la Encyclopedia of Bioethics, de 1995.

En consecuencia, la odontoestomatología, como parte de las ciencias de la salud, interviene y se enriquece con la bioética humanizando su praxis y relacionándose transdisciplinariamente.

El odontólogo, como efector sanitario, responsable de la salud oral del paciente y, por extensión, de la comunidad a la que pertenece, no debe estar ajeno a los fundamentos, valores, principios y metodología que la bioética propone(3), ya que éstos le posibilitarán un accionar profesional integrador de mayor calidad (bioética sanitaria o bioética clínica)(4).

La bioética, entendida como ética aplicada y normativa(5), abarca un campo muy amplio. Un aspecto es la relación médico/odontólogopaciente, interpretando a la luz de los valores éticos las características de esta relación, de modo que sea beneficiosa para ambas partes, sobre todo para la más vulnerable que es el paciente. Por extensión, a partir de esta relación clínica de dos individuos, la bioética como disciplina interviene en lo social, en la justicia sanitaria, en la asignación de recursos, investigación, educación para la salud, entre otras áreas, humanizando las profesiones del arte de curar.

Los conocimientos, habilidades y, sobre todo, actitudes tendrán más posibilidades de desarrollarse si se tienen como objetivo desde los comienzos de la formación de grado. Es en los primeros contactos con docentes, autoridades, compañeros y, fundamentalmente, pacientes donde se desarrollan normas de conducta éticamente correctas, de la mano de la fundamentación téorica que proporciona la bioética, dentro de otras asignaturas básicas o independiente de ellas, de acuerdo con la estrategia empleada por la institución educativa. Así se podrán entender en forma concreta la ética de las instituciones, de la relación clínica, de la investigación, y también la deontología.

Según Beauchamp y Childress(6) de la éticamente correcta relación sanitario-paciente, o investigador-sujeto de investigación, surgen principios y reglas, como la veracidad, buen uso de la información, confidencialidad, intimidad, entre otros, que el alumno aplica cuando comienza a interrogar a su primer paciente para confeccionar su historia clínica. Si bien se considera tácito el consentimiento informado para un examen odontológico de rutina, es importante que el alumno sepa de ello antes de ponerlo en práctica y, más adelante, cuál es el mejor tratamiento para ese paciente no sólo desde el punto de vista clínico, sino también desde el humano. Vale citar este sencillo ejemplo de un primer paso, como es el interrogatorio al paciente, para destacar que todo acto médico-odontológico es también un acto moral y merece ser considerado como tal. Al considerar la relación investigador-sujeto de investigación, valen las mismas pautas en cuanto a la ética de la investigación en seres humanos y, por lo tanto, estas pautas internacionales deben ser transmitidas tempranamente, de modo de introducir también al alumno en este tema.

\section{Metodología empleada}

- Búsqueda en la web de la información proporcionada por la oferta educativa del Estado argentino, a través de las páginas de las 
universidades existentes en el país que presentan en el grado académico la carrera de odontología.

- Análisis de los currículos o planes de estudio encontrados, buscando la presencia de la bioética, como asignatura o materia, en el desarrollo del cursado de la carrera y/o de las variantes que aparecieron.

- Se realizaron entrevistas con autoridades, docentes y alumnos en las facultades existentes en Mendoza, sin esquema previo.

- Se contactaron integrantes de universidades e instituciones nacionales y extranjeras: Círculo Argentino de Odontología, CONAMED de México y Universidad Javeriana de Colombia.

- Análisis de contenido de textos y artículos afines.

\section{Diagnóstico de situación}

Del análisis descrito se identifica la siguiente inserción de la bioética en los planes de estudio de odontología encontrados:

La bioética, como materia o actividad curricular, se dicta solamente en dos universidades de las doce encontradas que ofrecen el título de odontólogo, esto corresponde al 16,6\% de los casos. En las restantes diez se dicta dentro de los currículos como una unidad, eje temático o tema aislado en las materias afines, a saber: Odontología Legal y/o Forense, Deontología, Odontología Social, Odontología Sanitaria, Ejercicio Profesional, Ética, Psicología, Introducción a la Odontología Legal, Historia de la Odontología, etc. Estas asignaturas se presentan en distintos ciclos de los planes de estudios y varían en las distintas facultades y/o escuelas de odontología.
Otra forma de presentación es, dentro del currículo o en paralelo, como seminario o ta1ler. Extracurricularmente, además, toma la forma de seminario, taller o ateneo para estudiantes y docentes de odontología o, en otros casos, con la participación de integrantes de otras facultades. Finalmente, se presenta como actividad de posgrado, como curso o maestría.

Podría considerarse actualmente mínima la expresión de la bioética como materia curricular en la formación del odontólogo en el proyecto educativo argentino.

En la región de Cuyo encontramos dos universidades, una estatal (Universidad Nacional de Cuyo) y otra privada (Universidad de Mendoza), donde existen facultades de odontología. La bioética se presenta como tema en materias afines o como seminario. En entrevistas con autoridades y docentes de ambas facultades se observa gran interés, entendiendo que ampliar el dictado de la bioética, bajo cualquier estrategia, sería beneficioso para el estudiante y también para la institución educativa.

En entrevistas con los estudiantes del último año se aprecia desconocimiento del tema, pero interés en aprender de qué se trata. La concurrencia a los cursos o seminarios optativos es escasa en este momento; sin embargo, existen puntos de desarrollo interesantes que logran mayor concurrencia, como el caso de los ateneos, con debate de estudiantes y profesores de distintas facultades en la Universidad de Mendoza, que además se publican en su página web(7), y el del seminario que se imparte en la Facultad de Odontología de la Universidad Nacional de Cuyo.

\section{¿Debe incluirse en la estructura curricular? ¿Por qué?}

El debate sobre si la bioética debe dictarse dentro o fuera del currículo universitario y si 
realmente es relevante en la formación de los profesionales radica en que debemos resolver si la bioética es o no un conocimiento indispensable para el efector de salud.

Paulina Taboada(8) expresa que la ética médica responde a una experiencia humana básica y universal y no meramente a la fuerza de una moda. La ética es una manera de valorar acciones humanas relacionadas con los dilemas éticos que plantea el ejercicio de la medicina contemporánea. Eduardo Rodríguez(9) sostiene que el médico, y todo profesional relacionado con el arte de curar, debe ser educado en ética médica, formando su carácter moral. Para generar conocimientos, habilidades y actitudes, además de este carácter moral referido a lo aptitudinal, se requiere un proceso educativo gradual, tanto en contenidos como en tiempo de cursado, que acompañe al alumno en sus materias a lo largo de todo el currículo.

Desde su etapa de estudiante, el odontólogo se enfrenta diariamente con dilemas y su formación ética le aportará un enfoque más humanitario y humanístico. Esta formación adquiere una mayor eficacia dentro de los primeros años de estudio y no en el posgrado.

También es interesante destacar que la bioética, como materia básica o clínica, debería englobar conocimientos generales y prácticos de manera dinámica y aplicable, para interesar a un tipo de alumno que cursa una carrera eminentemente biotécnica.

Se trata de introducir tempranamente al alumno en el nexo entre dos mundos: valores éticos y hechos biológicos, e invitarlo a transitar este puente como espacio de reflexión, promoviendo el trabajo interdisciplinario.

Nuevas demandas profesionales que justifican la inclusión de la asignatura de bioética en la enseñanza de pregrado:
- Conocer los límites éticos de su praxis respecto de los avances de la tecnociencia.

- Reconocer los nuevos problemas que se plantean a raíz del avance de la tecnociencia y resolverlos con la mirada bioética.

- Entender el nuevo código de la relación odontólogo-paciente, reconociendo al paciente como persona en su dignidad, respetando su autonomía a través del consentimiento informado y la creciente horizontalización de esta relación.

- Conocer y aplicar en investigación sobre seres humanos normas vigentes de respeto por la vida y salud.

- Introducir en las asociaciones profesionales el conocimiento y respeto por los principios y valores de la bioética.

- Distribuir con justicia la asignación de recursos en el área de gestión en salud.

- Realizar auditoría en salud con fundamentos bioéticos.

- Participar activamente en los comités de bioética hospitalarios e institucionales, conociendo los mecanismos de toma de decisiones con una actitud de diálogo, apertura, tolerancia y juicio crítico.

- Promover la existencia de comités de bioética y ética de la investigación.

- Entender la interdisciplina como una necesidad real.

- Aplicar y transmitir el código de ética profesional.

- Conocer la situación actual en odontoestomatología legal y forense. 
- Conocer de bioseguridad en odontoestomatología, como normas sanitarias para evitar la transmisión de infecciones y la regulación de desechos tóxicos.

Estas nuevas demandas profesionales abarcan todos los ámbitos del quehacer profesional, generando así un nuevo perfil del egresado:

\section{Tras la formación en bioética, el odontólogo deberá ser capaz de:}

- Rejerarquizar la relación odontólogo-paciente centrada en la persona y su dignidad.

- Resolver en forma argumentada los conflictos éticos que aparezcan en su práctica profesional.

- Participar en forma significativa, con apertura y juicio crítico, en comités de bioética y/o de investigación.

- Respetar los límites éticos en investigación.

- Poseer juicio crítico para decidir en el ámbito de las asociaciones profesionales, deontológicas, gremiales y/o gubernamentales, con elementos de argumentación basados en los fundamentos bioéticos.

- Trabajar en equipos inter y transdisciplinarios en forma civil, pluralista, racional y procedimental.

- Entender los avances de la tecnociencia en beneficio del paciente y de la sociedad.

- Divulgar estos nuevos principios a pacientes, colegas, instituciones y alumnos.

La ubicación temporal de la asignatura debería responder a la complejidad de los contenidos que el alumno está cursando, vale decir, conjuntamente con las materias básicas en el segundo o tercer año se dictaría "bioética fun- damental" y, en el último semestre o año, "bioética clínica” con las especialidades clínicas.

Otra posibilidad de ubicación temporal sería atravesar con el tema todas las especialidades o materias afines (como interdisciplina, en unidades o ejes temáticos, en forma fragmentada o aplicada a los contenidos de cada una de ellas). O bien, una combinación de las anteriores posibilidades.

La formación en bioética comenzaría con los conceptos básicos, fundamentos de la ciencia, su historia y evolución, con el fin de lograr un lenguaje básico de comunicación, conjuntamente con las materias generales del currículo. Luego, retomaría su lugar en el último semestre de la carrera, para refrescar estos conocimientos y relacionarlos con las especialidades que se cursan en ese momento, aportando una mirada ética desde sus principios y valores, pudiendo los alumnos extraer ejemplos de su propia práctica clínica.

En este nivel de la carrera, habiendo tomado contacto con las especialidades clínicas y hospitalarias, el alumno puede reconocer los conflictos éticos, identificar los principios que aparecen en ellos, realizar un análisis crítico y aplicar conceptos de interdisciplina.

\section{Conclusiones}

En Argentina se observa la actual ausencia de la bioética en los planes de estudio de odontología, entendida como una asignatura del currículo que exprese la necesidad de reflexionar sobre los constantes dilemas éticos que se presentan en la praxis diaria del profesional odontólogo.

Esta evidencia merece ser considerada y ampliada, dando lugar a una nueva investigación detallada en cada universidad argentina que tenga a su cargo formación de odontólo- 
gos. Se deben atender las distintas necesidades de cada región del país, las opiniones de quienes imparten y reciben enseñanza y las características de la sociedad a quien va dirigida la acción de salud.
Según lo señala Pablo S. Lorda, todo problema clínico, hasta el aparentemente más sencillo, como recetar una aspirina, encierra un dilema ético(10); podríamos inferir que todo acto médico-odontológico (sanitario) encierra un aspecto ético que atender y conocer.

\section{Referencias}

1. Potter VR. Bioethics: Bridge to the future. New Jersey: Prentice-Hall Inc, Englewood Cliffs; 1971.

2. Reich WT. Encyclopedia of Bioethics. New York: Simon \& Schuster; 1995.

3. Gracia D. La deliberación moral: el método de la ética clínica. En: Gracia D, Júdez J (coord.) Bioética para clínicos. Barcelona: Med Clin: 2001.

4. Gracia D. Fundamentación y enseñanza de la bioética. Santafé de Bogotá: El Búho; 1998: 30.

5. Sgreccia E. Manual de Bioética. México: Diana; 1996: 135.

6. Beauchamp TL, Childress JF. Principios de Ética Biomédica. Barcelona: Masson S.A. 1999.

7. Ateneo de Bioética. Universidad de Mendoza. [Sitio en Internet] Disponible en www.um.edu.ar

8. Taboada P. Ética clínica: principios básicos y modelo de análisis. Boletín de la Escuela de Medicina, Pontificia Universidad Católica de Chile, 1998; 27(1).

9. Rodríguez E. Relación médico-paciente y práctica médica. En: Lolas F, (ed.) Diálogo y cooperación en salud. Diez años de bioética en la OPS. Santiago de Chile: Programa Regional de Bioética OPS/OMS; 2004: 70-71.

10. Lorda PS. Bioética para Clínicos. Capacidad de los pacientes para la toma de decisiones. Rev Española de Med Clin 2001; 117(11):419-426.

Recibido: 03 de enero de 2006

Aceptado: 31 de enero de 2006 